\title{
Generation of Coherent 19- and 38-nm Radiation at a Free-Electron Laser Directly Seeded at $38 \mathrm{~nm}$
}

\author{
S. Ackermann, ${ }^{1,2}$ A. Azima, ${ }^{1,5,6}$ S. Bajt, ${ }^{2}$ J. Bödewadt, ${ }^{1,5}$, F. Curbis, ${ }^{1, \dagger}$ H. Dachraoui, ${ }^{2}$ H. Delsim-Hashemi, ${ }^{2}$ \\ M. Drescher, ${ }^{1,5,6}$ S. Düsterer, ${ }^{2}$ B. Faatz, ${ }^{2}$ M. Felber, ${ }^{2}$ J. Feldhaus, ${ }^{2}$ E. Hass, ${ }^{1}$ U. Hipp, ${ }^{1}$ K. Honkavaara,${ }^{2}$ R. Ischebeck, ${ }^{4}$ \\ S. Khan, ${ }^{3}$ T. Laarmann, ${ }^{2,6}$ C. Lechner, ${ }^{1}$ Th. Maltezopoulos, ${ }^{1,5}$ V. Miltchev,${ }^{1}$ M. Mittenzwey, ${ }^{1}$ M. Rehders, ${ }^{1,5}$ \\ J. Rönsch-Schulenburg, ${ }^{1,5}$ J. Rossbach, ${ }^{1,5}$ H. Schlarb, ${ }^{2}$ S. Schreiber, ${ }^{2}$ L. Schroedter, ${ }^{2}$ M. Schulz, ${ }^{1,2}$ S. Schulz, ${ }^{2}$ \\ R. Tarkeshian, ${ }^{1, \$}$ M. Tischer, ${ }^{2}$ V. Wacker, ${ }^{1}$ and M. Wieland ${ }^{1,5,6}$ \\ ${ }^{1}$ Department of Physics, University of Hamburg, 22761 Hamburg, Germany \\ ${ }^{2}$ Deutsches Elektronen-Synchrotron DESY, 22607 Hamburg, Germany \\ ${ }^{3}$ Technische Universität Dortmund, 44227 Dortmund, Germany \\ ${ }^{4}$ Paul Scherrer Institut, 5323 Villigen, Switzerland \\ ${ }^{5}$ Center for Free-Electron Laser Science CFEL, DESY, 22607 Hamburg, Germany \\ ${ }^{6}$ The Hamburg Centre for Ultrafast Imaging CUI, 22761 Hamburg, Germany \\ (Received 4 March 2013; published 9 September 2013)
}

\begin{abstract}
Initiating the gain process in a free-electron laser (FEL) from an external highly coherent source of radiation is a promising way to improve the pulse properties such as temporal coherence and synchronization performance in time-resolved pump-probe experiments at FEL facilities, but this so-called "seeding" suffers from the lack of adequate sources at short wavelengths. We report on the first successful seeding at a wavelength as short as $38.2 \mathrm{~nm}$, resulting in GW-level, coherent FEL radiation pulses at this wavelength as well as significant second harmonic emission at $19.1 \mathrm{~nm}$. The external seed pulses are about 1 order of magnitude shorter compared to previous experiments allowing an ultimate time resolution for the investigation of dynamic processes enabling breakthroughs in ultrafast science with FELs. The seeding pulse is the 21st harmonic of an 800-nm, 15-fs (rms) laser pulse generated in an argon medium. Methods for finding the overlap of seed pulses with electron bunches in spatial, longitudinal, and spectral dimensions are discussed and results are presented. The experiment was conducted at FLASH, the FEL user facility at DESY in Hamburg, Germany.
\end{abstract}

DOI: 10.1103/PhysRevLett.111.114801

PACS numbers: 41.60.Cr, 42.55.-f, 42.65.Ky

The invention of high-gain free-electron lasers (FEL) and the realization of FEL facilities producing ultrashort, intense radiation pulses from the extreme ultraviolet (EUV) to the hard $\mathrm{x}$-ray spectral range have opened completely new opportunities for studies of dynamical processes at the atomic level. Such light sources, among them the Free-electron LASer in Hamburg (FLASH) [1], the FERMI@Elettra facility [2], the Linear Coherent Light Source (LCLS) [3], or the SPring-8 Angstrom Compact free-electron LAser (SACLA) [4] have the ability to generate $\mathrm{x}$-ray pulses with up to $10^{13}$ photons within a pulse duration of only a few tens of femtoseconds $[1,3]$. However, compared to conventional lasers, the degree of temporal, i.e., longitudinal, coherence of an FEL is rather low when operated in the so-called self-amplified spontaneous emission (SASE) mode [5,6]. The reason is found in the stochastic nature of the spontaneous undulator radiation which is used to induce the FEL amplification process. Different schemes have been proposed in the past to overcome this limitation [7-13]. Most of them use external lasers either to manipulate the position-andmomentum phase-space distribution of the electrons $[9,13]$ or to directly act as a seed for the FEL amplification process (direct FEL seeding) [10]. The arrival time of the femtosecond FEL pulses fluctuates from pulse to pulse relative to a synchronized external optical signal. This fluctuation is caused by arrival-time variations of the electron bunches at the undulators, the SASE process itself, and the limited ability to synchronize the external laser pulse to the electron bunch [14-16]. In order to achieve highest time resolution for dynamic studies in opticalpump-FEL-probe schemes, one could either reduce the arrival-time fluctuations below the duration of the pump and probe pulses or measure the arrival time of the FEL pulses on a shot-to-shot basis [17,18] and sort the data accordingly [19-21]. A common source of optical laser and EUV pulses for pump-probe experiments will greatly improve the time resolution due to the intrinsic synchronization which is one of the main advantages of an externally seeded FEL. In combination with short seed pulses, it enables, e.g., detailed studies of important ultrafast dynamics at work in molecular chemistry [22]. Up-conversion of photon frequencies by means of nonlinear high-harmonic generation (HHG) in gases [23] allows us to create coherent radiation in the EUV spectral range which can then be used to directly seed the FEL process. An extension of this approach towards shorter wavelengths, however, represents a major challenge, owing to increasing required 
seed intensities [24] together with falling HHG efficiency [25]. The first direct FEL seeding with an HHG source was demonstrated at the SPring-8 Compact SASE Source test accelerator (SCSS) [26] in 2008 [27] at a $160 \mathrm{~nm}$ wavelength (fifth harmonic of a Ti:sapphire laser system). Later, the 13th harmonic at $61 \mathrm{~nm}$ was used for seeding [28]. Further experimental studies on direct FEL seeding with HHG radiation have been performed at SPARC [29]. The present Letter reports on the first successful operation of direct FEL seeding at a wavelength as short as $38 \mathrm{~nm}$ with seed pulse durations below $15 \mathrm{fs}$ (rms).

The seeding experiment is performed at the FEL facility FLASH at DESY [30,31]. The electron beam is generated in a normal-conducting radio frequency (rf) photocathode electron gun running at $1.3 \mathrm{GHz}$ and is injected into a superconducting linear accelerator equipped with two bunch compressor sections. For the seeding experiment, the machine was operated in single-bunch mode, i.e., one electron bunch with a repetition rate of $10 \mathrm{~Hz}$. As illustrated in Fig. 1, the injection of the EUV beam for seeding takes place at the last dipole magnet of the electron beam collimation system. Electron beam and seed radiation are then collinearly transported into the seeding undulator [see (7) in Fig. 1]. Note that the FLASH SASE undulator is a different device further downstream and will not be further mentioned in this text. Additional information about the setup can be found in Ref. [32] and references therein.

The laser-driven high-harmonic source to generate the EUV radiation for seeding is described in detail in Ref. [33]. It is based on a Ti:sapphire laser system delivering pulses with an energy of $18 \mathrm{~mJ}$ within a pulse duration of $15 \mathrm{fs}$ (rms) and a repetition rate of $10 \mathrm{~Hz}$ [near-infrared (NIR) laser in Fig. 1]. The laser oscillator of the seed laser system is electronically synchronized to the $1.3-\mathrm{GHz}$ master clock of the FLASH facility [34] with a typical synchronization fluctuation of $50 \mathrm{fs}$ (rms). NIR laser pulses are loosely focused with an $f=1.5 \mathrm{~m}$ mirror in a pulsed argon medium. For the data shown in this Letter, the HHG source had a single-shot pulse energy at the 21 st harmonic of $9 \mathrm{~nJ}$ with a stability of $10 \%$ (rms). The EUV beam is injected into the seeding undulator by remotely controlled mirrors to establish the spatial laser-electron overlap. The mirror coatings allow an optimal transport of the 21 st harmonic while other wavelengths are suppressed significantly. Several diagnostic stations between the undulator modules are capable of measuring the transverse beam profile and position of the EUV and the electron beam (see Fig. 1). The relative timing between electron bunches and seed pulses is measured in two ways. First, synchrotron radiation from a five-period electromagnetic undulator [see (5) in Fig. 1] is sent into a timing diagnostic setup together with the remaining infrared light from the HHG drive laser pulse. The setup comprises a photomultiplier and a streak camera which allows us to set the temporal overlap of electron and photon pulses with approximately \pm 2 -ps precision. To determine the relative delay to the sub-ps level, the coherent enhancement of optical undulator radiation of a second electromagnetic undulator (6), which is tuned to the second harmonic at $400 \mathrm{~nm}$, is monitored. This enhancement is initiated through an interaction of the NIR laser with the electron bunch in the first electromagnetic undulator (5), causing a periodic modulation of the electron energy. A subsequent magnetic chicane (8) converts this energy modulation into a density modulation, leading to the coherent emission of radiation at $400 \mathrm{~nm}$ [35].

The seeding undulator section consists of a variable-gap planar undulator system [see (7) in Fig. 1] comprising three 2-m-long insertion devices with a period of $31.4 \mathrm{~mm}$ and one 4-m-long undulator with a period of $33 \mathrm{~mm}$ [36]. To separate the FEL radiation generated in the undulators from the electron beam, a mirror triplet is inserted behind the undulators, reflecting the photon beam into dedicated diagnostic beam lines with calibrated energy monitors and spectrometers, while the electrons are deflected by a short magnetic chicane to bypass the mirrors [see (9) in Fig. 1] [37].

Table I lists typical parameters for the electron and the seed beam. The electron beam current distribution is

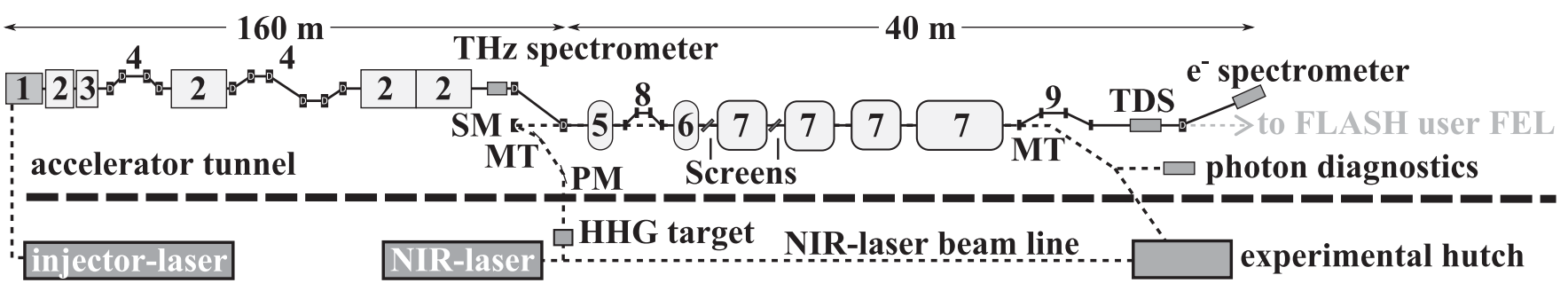

FIG. 1. Schematic layout of the FLASH facility. The beam direction is from left to right. The electron beam is generated in a laserdriven rf gun photo injector (1) and accelerated up to $1.25 \mathrm{GeV}$ in a superconducting linear accelerator (2) with a frequency of $1.3 \mathrm{GHz}$. A 3.9-GHz rf resonator module (3) controls the longitudinal bunch charge distribution. Two magnetic bunch compressors (4) allow us to generate high-peak-current electron bunches. Two short electromagnetic undulators $(5,6)$ are used for diagnostic purposes. Small magnetic chicanes $(8,9)$ allow us to steer the electron beam around mirrors for photon diagnostics. The HHG drive laser and the HHG target are located outside of the accelerator tunnel. One plane mirror (PM), a mirror triplet (MT), and one spherical mirror (SM) are used to inject the EUV light into a 10-m-long series of variable-gap seeding undulators (7). Further downstream, the electron beam is sent through a transverse deflecting structure (TDS) and to an electron spectrometer for diagnostic reasons, or to the SASE FEL of the FLASH user facility. 
TABLE I. Summary of the electron and seed laser beam parameters at the seeding undulator entrance.

\begin{tabular}{lc}
\hline \hline Electron beam & Value \\
\hline Energy & $700 \mathrm{MeV}$ \\
Energy spread (rms) & $2 \times 10^{-4}$ \\
Emittance & $1.7 \mathrm{~mm} \mathrm{mrad}$ \\
Bunch charge & $0.5 \mathrm{nC}$ \\
Peak current & $1 \mathrm{kA}$ \\
Power gain length & $0.70 \mathrm{~m}$ \\
Average beam size (rms) & $170 \mu \mathrm{m}$ \\
\hline Seed beam & Value \\
\hline Pulse energy (at source) & $9 \mathrm{~nJ}$ \\
Pulse energy stability (rms) & $10 \%$ \\
Central wavelength & $38.2 \mathrm{~nm}$ \\
Spectral bandwidth (rms) & $0.09 \mathrm{~nm}$ \\
Pulse duration (rms) & $<15 \mathrm{fs}$ \\
Average beam size (rms) & $280 \mu \mathrm{m}$ \\
\hline \hline
\end{tabular}

measured with a fast longitudinal profile monitor based on single-shot wavelength-resolved $\mathrm{THz}$ detection [38]. The undulators are set to a gap value corresponding to approximately $38 \mathrm{~nm}$ for the given electron beam energy of $700 \mathrm{MeV}$. For the precise spectral overlap, the HHG and FEL spectra are measured with the same spectrometer. Typical single-shot SASE spectra, the average over 100 shots of these spectra, and the HHG reference spectrum are shown in Fig. 2(c). In order to verify that the first undulator module, at which the seeding takes place, contributes significantly to the FEL gain, the undulator gap is scanned. Measuring the gain curve of the FEL assures that the FEL amplifier for the HHG radiation is set up correctly and results in a power gain length of $(0.70 \pm 0.04) \mathrm{m}$. Therewith, the average pulse energy $\left\langle E_{\mathrm{SASE}}\right\rangle$ of the seeded FEL was measured to be $(1.3 \pm 0.5) \mu \mathrm{J}$. To establish the transverse photon-electron overlap within the first undulator module, the EUV seed and the electron beam profiles are measured on cerium-doped YAG screens in front of the first undulator module [Fig. 2(a)] and at the exit [Fig. 2(b)]. Using the steering capabilities of the seed injection beam line and the corrector magnets, an iterative procedure is used to overlap both beams on these screens without disturbing the FEL amplification performance. The temporal overlap between the laser pulses and the electron bunches is established to the sub-ps level using the timing diagnostics described earlier. To this end, the delay between both pulses is scanned by electronically changing the phase of the synchronization signal for the laser oscillator. The delay is scanned in steps of $100 \mathrm{fs}$ within a range of \pm 2 ps. Dedicated software analyzes the FEL pulse energy and the data from the spectrometer on a shot-to-shot basis to discriminate the seed signal from unseeded SASE amplification. If one value within a set of about 30 machine parameters is outside of the nominal range for one shot, the software disregards this shot and proceeds until a fixed number of well-defined samples at the same time delay is

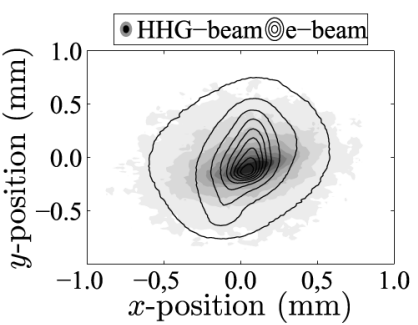

(a)

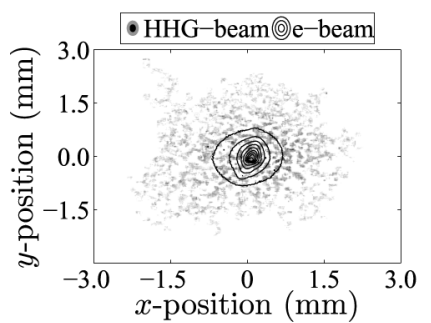

(b)

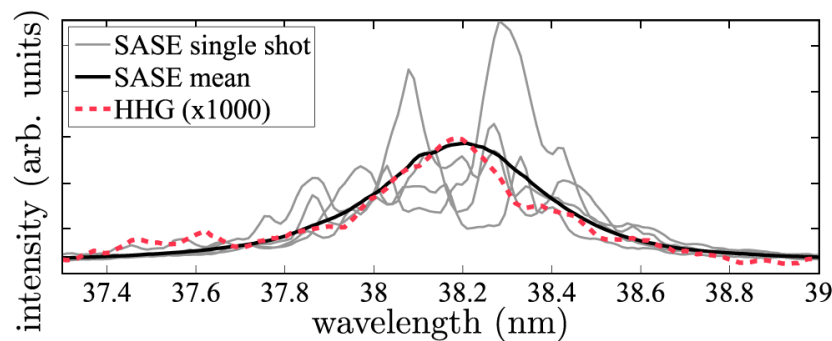

(c)

FIG. 2 (color online). Illustration of spatial and spectral overlap. The upper panels show the normalized spatial distribution of the EUV beam (filled contours) and the electron beam (black contours) (a) in front of and (b) after the first undulator module, respectively (see also Fig. 1). Note the different scales. In (c), typical single-shot SASE spectra (solid grey lines), an average SASE spectrum (solid black line), and an HHG spectrum of 20 pulses accumulated and multiplied by 1000 (dotted red line) are plotted.

reached. To clearly identify radiation from the seed process, the seed laser is toggled on and off every two seconds. In total, this procedure assures that possible fluctuations or drifts of any subsystem which would change the SASE pulse energy distribution can be excluded.

Figure 3(a) shows the result of two delay scans with a total scan range of 4 ps. For every delay, 200 FEL pulses were evaluated. In the range between $t=-0.5$ and $0.5 \mathrm{ps}$, a strong positive correlation between the seed input and the FEL output signal [Fig. 3(b)] is observed, demonstrating successful seeding. The width of the curves in Fig. 3 is dominated by the relative laser-electron arrival-time variations, since no active compensation for the arrival time of the laser pulses was used. An alternative analysis of the same data set is presented in Fig. 4. Without HHG pulses, the FEL generates only SASE radiation and a histogram of the FEL pulse energies assumes the shape of a Gamma probability distribution [39], plotted as a solid line in Figs. 4(a) and 4(b). The histograms in these figures comprise the data from Fig. 3 with an activated seed laser. Values with energy contrast $>2.5$ are accumulated in the rightmost $E /\left\langle E_{\mathrm{SASE}}\right\rangle$ bin. Figure 4(a) shows the data in the time range from $t=-0.4$ to $0.4 \mathrm{ps}$, while the histogram for the range from $t=-1.7$ to -0.9 ps is plotted in Fig. 4(b). Ideally, for each bunch being seeded without any source of spatiotemporal jitter, a probability distribution reflecting the statistics of the HHG pulse energies is expected. However, during the time of this experiment, the arrivaltime spread of the electron bunches has been measured to be $500 \mathrm{fs}$ (peak to peak), which is $25 \%$ larger than the typical 


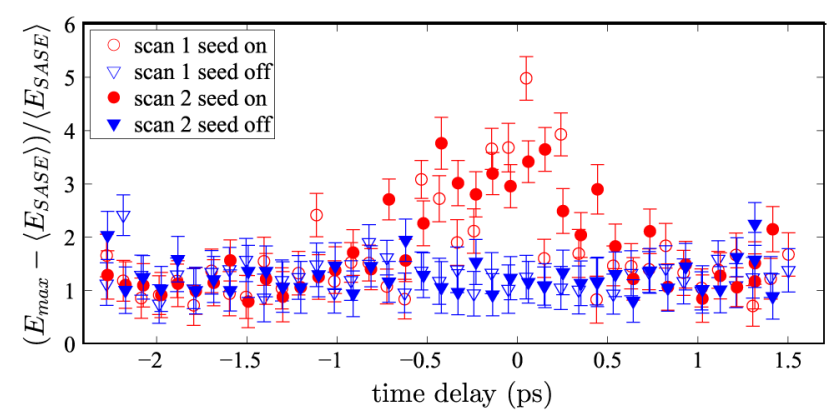

(a)

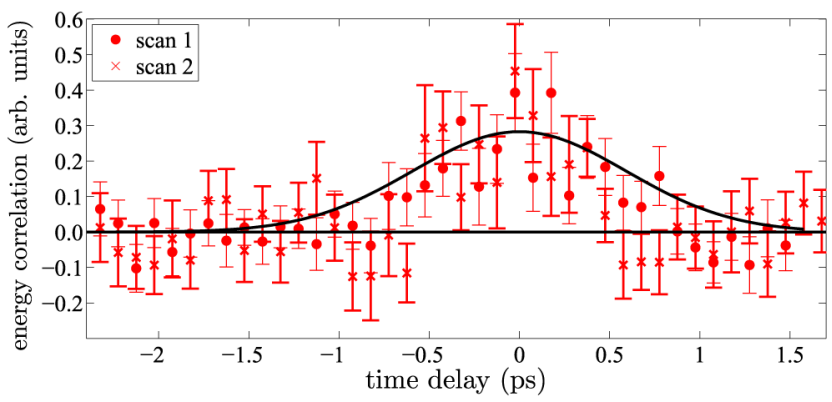

(b)

FIG. 3 (color online). Results of time delay scans. (a) Contrast of the maximum pulse energy and the averaged pulse energy of SASE for each time delay as obtained in two independent scans. (b) Correlation data of the EUV seed pulse energy and the output energy for both scans. Error bars indicate 1 standard deviation of statistical fluctuations.

spread in a single-bunch operation [40]. Therefore, the optimum temporal overlap is achieved only for a fraction of the bunches and the high-energy events are smeared out to lower energy values. Evidently, about $5 \%$ of the shots have an energy contrast above 2.5 within the range with temporal overlap [Fig. 4(a)]. These events are clearly not compatible with the SASE distribution. In contrast, without any temporal overlap, no significant increase of events with high energy contrast is visible [Fig. 4(b)].

Ultimately, the effect of seeding can be seen in the spectral domain. Besides the enhancement of the spectral intensity at the fundamental wavelength, a significant increase of the signal is measured at the second harmonic of the seeded FEL, as shown in Fig 5. A maximum contrast of 36 can be seen between the highest events with seeding, as compared to the mean intensity without seeding.

Furthermore, the gap of the first undulator module as well as the spatial position of the EUV beam were scanned around the nominal overlap region. In all dimensions, seeding is clearly verified and is in good agreement with the measured spectral bandwidth and beam sizes, respectively.

A transverse mode selection takes place during the FEL gain process due to diffraction. For our parameters, we conclude that the gain of the $\mathrm{TM}_{00}$ mode exceeds all others such that a high degree of transverse coherence exists at the exit of the undulator [41]. Furthermore, a high degree of longitudinal coherence can be concluded from the fact that the EUV seed pulse duration is significantly shorter than

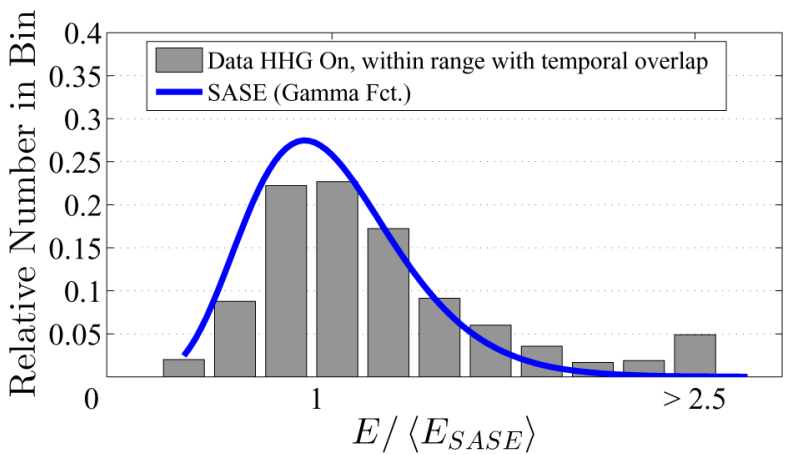

(a)

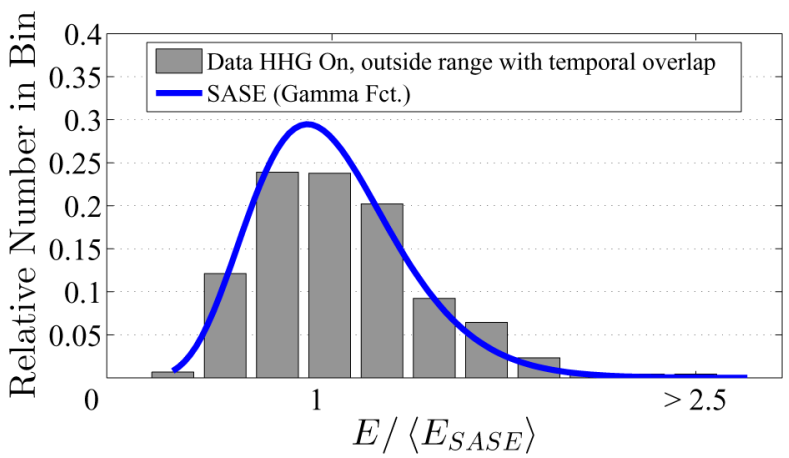

(b)

FIG. 4 (color online). Impact of seeding on the pulse energy distribution (a) within the overlap time range and (b) for a time delay of $>0.5 \mathrm{ps}$ between the electron bunch and HHG seed pulse. Values with energy contrast $>2.5$ are accumulated in the rightmost $E /\left\langle E_{\mathrm{SASE}}\right\rangle$ bin.

$15 \mathrm{fs}$ (rms) [33], while the FEL coherence time is $7.4 \mathrm{fs}$ (rms) for our parameters [42]. This conclusion is supported by Ref. [43], where the predominance of a nearly single (thus coherent) longitudinal SASE mode was demonstrated for a sufficiently short electron bunch which has been corroborated by numerical simulation using the code GENESIS 1.3 [44].

In this Letter, we have reported on the first demonstration of direct FEL seeding at $38 \mathrm{~nm}$. We have observed a maximum spectral contrast between seeded and unseeded

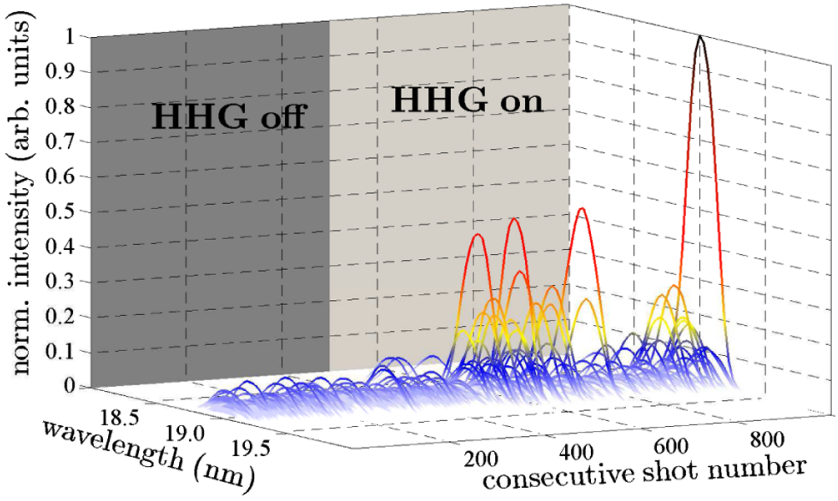

FIG. 5 (color online). 1000 consecutive spectra of the second harmonic of the seeded FEL with (HHG on) and without (HHG off) seeding. 
FEL pulses of 36. Direct seeding has been observed reproducibly and studied under variation of various parameters. Because of the high electron peak current of $1 \mathrm{kA}$, the measured power gain length is much shorter compared to previous HHG seeding experiments, which proves that HHG seeding is not prejudiced by effects which come along with high-current electron beams [45]. The fraction of effectively seeded bunches will be increased in the future by applying the intratrain longitudinal feedback system [40]. A further important step will be the realization of two-color cross-correlation experiments $[18,46]$ providing information on both spectral amplitude and the phase of the amplified light wave. These experiments will reveal to what extent the FEL process preserves the favorable pulse properties of HHG-generated EUV pulses, which is an indispensable precondition for coherent control of correlated electronic and nuclear dynamics with ionizing light fields on the ultrashort sub-50-fs time scales.

Without the help from many groups at DESY, the preparation and commissioning of all components of the seeding experiment would not have been conceivable. Their support is gratefully acknowledged. The authors would like to thank S. Reiche for helpful discussions. This work is supported by the Federal Ministry of Education and Research of Germany within FSP-301 under FKZ 05K10GU1 and the German Research Foundation within GrK 1355.

*joern.boedewadt@uni-hamburg.de

'Present address: MAX IV Laboratory, Lund University, Post Office Box 118, SE-221 00 Lund, Sweden.

Present address: Max Planck Institute for Physics, 80805 München, Germany.

[1] W. Ackermann et al., Nat. Photonics 1, 336 (2007).

[2] E. Allaria et al., Nat. Photonics 6, 699 (2012).

[3] P. Emma et al., Nat. Photonics 4, 641 (2010).

[4] T. Ishikawa et al., Nat. Photonics 6, 540 (2012).

[5] A. M. Kondratenko and E. L. Saldin, Part. Accel. 10, 207 (1980).

[6] R. Bonifacio, C. Pellegrini, and L. M. Narducci, Opt. Commun. 50, 373 (1984).

[7] J. Feldhaus, E. L. Saldin, J. R. Schneider, E. A. Schneidmiller, and M. V. Yurkov, Opt. Commun. 140, 341 (1997).

[8] A. A. Zholents, Phys. Rev. ST Accel. Beams 8, 040701 (2005).

[9] L.-H. Yu et al., Science 289, 932 (2000).

[10] L. DiMauro et al., Nucl. Instrum. Methods Phys. Res., Sect. A 507, 15 (2003).

[11] G. Geloni, V. Kocharyan, and E. Saldin, J. Mod. Opt. 58, 1391 (2011).

[12] J. Amann et al., Nat. Photonics 6, 693 (2012).

[13] G. Stupakov, Phys. Rev. Lett. 102, 074801 (2009).

[14] A. Cavalieri et al., Phys. Rev. Lett. 94, 114801 (2005).

[15] P. Radcliffe et al., Appl. Phys. Lett. 90, 131108 (2007).

[16] S. Cunovic et al., Appl. Phys. Lett. 90, 121112 (2007).
[17] Th. Maltezopoulos et al., New J. Phys. 10, 033026 (2008).

[18] I. Grguras et al., Nat. Photonics 6, 852 (2012).

[19] M. Harmand et al., Nat. Photonics 7, 215 (2013).

[20] M. Krikunova et al., New J. Phys. 11, 123019 (2009).

[21] M. Krikunova, Th. Maltezopoulos, P. Wessels, M. Schlie, A. Azima, M. Wieland, and M. Drescher, J. Chem. Phys. 134, 024313 (2011).

[22] K. Wynne and N. T. Hunt, Phys. Chem. Chem. Phys. 14, 6154 (2012).

[23] M. Ferray, A. L'Huillier, X. F. Li, L. A. Lompre, G. Mainfray, and C. Manus, J. Phys. B 21, L31 (1988).

[24] Z. Huang and K.-J. Kim, Nucl. Instrum. Methods Phys. Res., Sect. A 475, 59 (2001).

[25] E. L. Falcão-Filho, C.-J. Lai, K.-H. Hong, V.-M. Gkortsas, S.-W. Huang, L.-J. Chen, and F. X. Kartner, Appl. Phys. Lett. 97, 061107 (2010).

[26] T. Shintake et al., Nat. Photonics 2, 555 (2008).

[27] G. Lambert et al., Nat. Phys. 4, 296 (2008).

[28] T. Togashi et al., Opt. Express 19, 317 (2011).

[29] M. Labat et al., Phys. Rev. Lett. 107, 224801 (2011).

[30] K. Tiedtke et al., New J. Phys. 11, 023029 (2009).

[31] S. Schreiber et al., in Proceedings of the 2010 FreeElectron Laser Conference (JACoW, Malmö, 2010), p. 198.

[32] C. Lechner et al., in Proceedings of the 2012 FreeElectron Laser Conference (JACoW, Nara, 2012), p. 197.

[33] Th. Maltezopoulos et al., Appl. Phys. B, doi: 10.1007/ s00340-013-5571-6 (2013).

[34] M. Felber et al., in Proceedings of 2010 the Free-Electron Laser Conference (JACoW, Malmö, 2010), p. 544.

[35] P. M. Salén et al., in Proceedings of the 2009 FreeElectron Laser Conference (JACoW, Liverpool, 2009), p. 739.

[36] H. Delsim-Hashemi et al., in Proceedings of the 2010 International Particle Accelerator Conference (JACoW, Kyoto, 2010), p. 3114.

[37] F. Curbis et al., in Proceedings of the 2010 Free-Electron Laser Conference (JACoW, Malmö, 2010), p. 298.

[38] S. Wesch, B. Schmidt, C. Behrens, H. Delsim-Hashemi, and P. Schmüser, Nucl. Instrum. Methods Phys. Res., Sect. A 665, 40 (2011).

[39] E. L. Saldin, E. A. Schneidmiller, and M. V. Yurkov, Opt. Commun. 148, 383 (1998).

[40] S. Pfeiffer et al., in Proceedings of the 2012 International Particle Accelerator Conference (JACoW, New Orleans, 2012), p. 26.

[41] E. L. Saldin, E. A. Schneidmiller, and M. V. Yurkov, Opt. Commun. 97, 272 (1993).

[42] C. Behrens, N. Gerasimova, Ch. Gerth, B. Schmidt, E. A. Schneidmiller, S. Serkez, S. Wesch, and M. V. Yurkov, Phys. Rev. ST Accel. Beams 15, 030707 (2012).

[43] S. Reiche, P. Musumeci, C. Pellegrini, and J. B. Rosenzweig, Nucl. Instrum. Methods Phys. Res., Sect. A 593, 45 (2008).

[44] S. Reiche, Nucl. Instrum. Methods Phys. Res., Sect. A 429, 243 (1999).

[45] I. Zagorodnov and M. Dohlus, Phys. Rev. ST Accel. Beams 14, 014403 (2011).

[46] U. Frühling et al., Nat. Photonics 3, 523 (2009). 